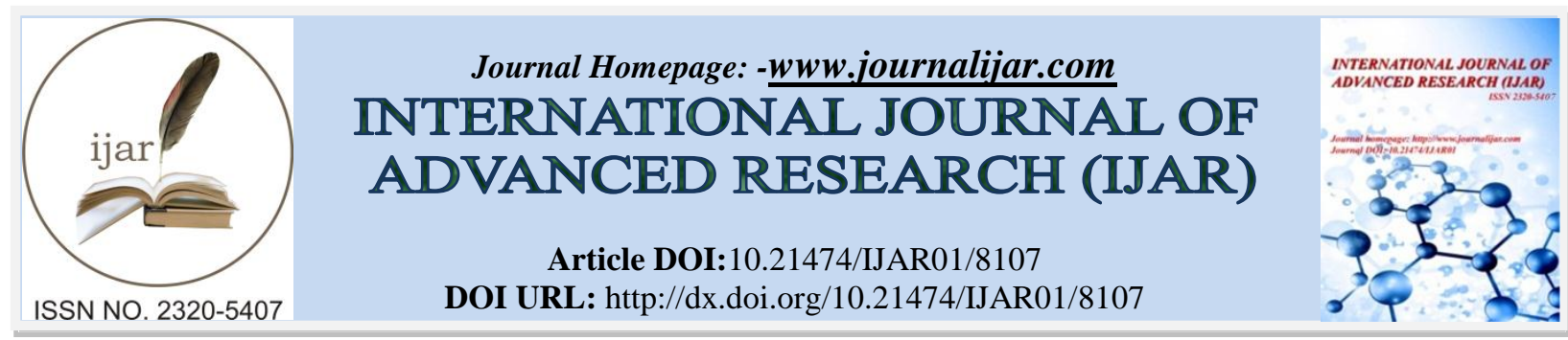

RESEARCH ARTICLE

\title{
COMPARISON BETWEEN EFFECT OF LONG AGONIST AND ANTAGONIST PROTOCOLS ON MICRO-RNA- 320 LEVEL IN FOLLICULAR FLUID DURING IVF- ICSI.
}

Mufeda Ali Jwad ${ }^{1}$, Ban Abbas ${ }^{2}$ and Ula Al-Kwaz ${ }^{3}$.

1. M.B.ch.B/ MSc. Applied Embryology. High Institute for Infertility Diagnosis \& Assisted Reproductive Technology / Al - Nahrain University.

2. M.B.ch. B/M.S.c, Ph.D Molecular Pathology, Medical collage/ Al- Nahrain University.

3. High Institute for Infertility Diagnosis \& Assisted Reproductive Technology.

\section{Manuscript Info}

(..........................

Manuscript History

Received: 01 October 2018

Final Accepted: 03 November 2018

Published: December 2018

Keywords:-

Mirna, stimulation protocol, ivf-icsi.

\section{Abstract}

Background:The natural cycle (unstimulated cycle) was used in first IVF trial which give birth of a life baby. However, it was soon recognized that the success rate of IVF in natural cycles was low, this may be due to the low number of oocytes retrieved. Ovarian stimulation using urinary gonadotropins was applied to deal with this problem, resulting in a significant increase in both the number of eggs retrieved, as well as the success rate of IVF. But in the same time the using of stimulation drugs may negatively affect oocyte and embryo quality which could diagnosed by using non invasive procedure like measurement of miRNA- 320 in follicular fluid.

Aim of study: To compare the effect of stimulation protocol on ICSI outcome using miRNA- 320 that reflects the embryo quality.

Materials and method: This comparative study was done on the embryos of seventy one infertile females who were undergoing Intracytoplasmic sperm injection (ICSI) treatment regardless to the presence or absence of previous trials. These patients were divided into two groups, 45 of them treated with agonist protocol and other 26 patients treated with antagonist protocol. Of the 71 oocytes, there were eight immature oocytes (3 at germinal vesicle $(\mathrm{GV})$ stage, 5 at metaphase I (MI) stage) and three abnormal oocytes, and these were excluded from the following ICSI treatment. Measurement of follicular fluid mi-RNA 320 (which is known biomarker of embryo quality) was done by PCR.

Results:The statistical analysis showed no significant correlation between miRNA- 320 levels in follicular fluid and age, BMI, number of dominant follicles, number of oocytes retrieved, number of metaphase I, metaphase II, germinal vesicle, abnormal oocytes and number of fertilized oocytes (2PN). The comparison between miRNA 320 levels in good and bad quality embryos showed highly significant $(\mathrm{p}<0.001)$ difference but no significant difference $(\mathrm{p}>0.05)$ was found between the different stimulation protocols.

Corresponding Author:-Mufeda Ali Jwad. Address:-M.B.ch.B/ MSc. Applied Embryology, Al - Nahrain University Al- Nahrain medical collage/ $\mathrm{Al}$ - Nahrain University. 
Conclusions:The stimulation protocol does not affect the level of miRNA which is molecular marker of embryo quality.

Copy Right, IJAR, 2018,. All rights reserved.

\section{Introduction:-}

The key factor for successful pregnancy is an embryo quality which involves the morphological characteristics and cleavage rates that are currently used for selecting and transferring embryos. So trials were done to find out predictive biomarkers in the follicular fluid that are associated with fertility related phenotypes, including follicle development, oocyte fertilization, embryo quality, and pregnancy outcomes (Feng etal., 2015).

Because the follicular fluid affects oocyte development, its composition has been investigated as a possible predictor of oocyte and embryo quality (Ledee et al.,2008; Cupisti et al., 2007)

Recently; it was discovered that micro- RNA (miRNAs) is one of the constituents of FF in human follicle. These molecules were suggested to be used as biomarkers of oocyte quality. Some of the recent studies confirmed the presence of miRNA in FF of human and animal models, but their origin and the role that they could perform inside ovarian follicles were not established exactly (da Silveira et al., 2012; Sohel et al., 2012).

Micro RNAs (miRNAs) are a class of small, non coding, endogenous RNAs whose mature products are approximately 21-25 nucleotides (nts) in length (Wahid et al., 2010), derived primarily from introns of coding genes in the mammalian genome ( $\mathrm{Su}$ et al., 2010). They play an important regulatory role in animals and plants by targeting specific mRNAs for degradation or translation repression. The regulation of miRNA is concerned in disease etiology and therapeutics (Kim 2005).

It was demonstrated that miR-320 were among the top 10 highest expressed miRNAs in follicular fluid (Diez-fraile et al. 2014). MiRNA- 320 plays an important role in embryo development potential in which higher expression level was demonstrated in embryos of good quality. The previous studies provided an evidence that miRNAs mainly miR320 in human follicular fluid might reflect and affect embryo quality through regulating multiple signaling pathways, including Wnt signaling pathway (Sonderegger et al., 2010).

\section{Materials and method:-}

This prospective comparative study included seventy one women who underwent ICSI at the infertility clinic of High Institute of Infertility Diagnosis and Assisted reproductive technologies/ Al- Nahrain University/ Baghdad/ Iraq, during the period from December 2015 until December 2016. Their age range was 20 to 43 years old. The infertility duration range 1-20 years with both primary and secondary infertility. Some of patients have their first IVF or ICSI attempt, while others had already undergone at least one cycle before.

These patients were stimulated by two different protocols, 45 of them treated with long agonist protocol and other 26 patients treated with antagonist protocol. The long agonist protocol and antagonist protocols are summarized in figure (1)

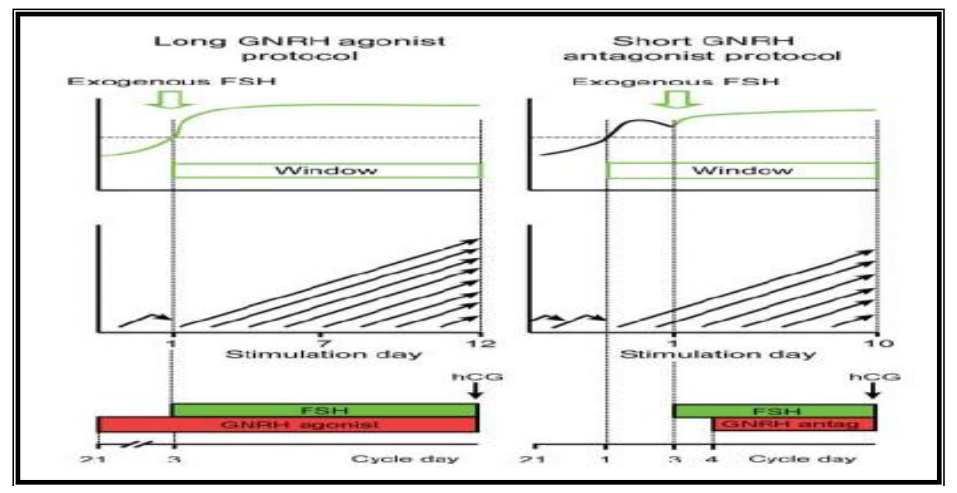

Figure1:- long GNRH agonist regimen; GNRH antagonist regimen. The window represents the duration of time at which FSH concentrations are above the threshold. Each arrow represents a developing follicle. Adapted from

(Macklon et al. (2006). 
All the 71 women, had a normal ovarian reserve, based on the serum AMH level and antral follicle count (AFC), evaluated at day 3 of the menstrual cycle. Oocytes were retrieved by transvaginal ultrasound-guided aspiration 3436 hours after hCG injection. For each patient, all follicles were aspirated without flushing media; cumulus-oocyte complexes were isolated. Oocyte retrieval was done under general or sometimes spinal anasthesia according to the patient response. Normal saline solution was used to clean vagina thoroughly. Aspiration was achieved using single lumen needle using aspiration media as a flushing medium. Aspiration pump pressure was set around $120 \mathrm{~mm} \mathrm{Hg}$.

A volume of $1 \mathrm{ml}$ follicular fluid from each isolated oocyte was centrifuged at $2800 \mathrm{~g}$ for $10 \mathrm{~min}$ at $4^{\circ} \mathrm{C}$. Then, supernatants were aspirated, eliminate cell debris and stored at $-40{ }^{\circ} \mathrm{C}$. The miRNA -320 expression profile of the follicular fluid was produced and used qRT-PCR to identify miRNA-320 which is associated with embryonic developmental potential.

\section{Micro RNA extraction:-}

Micro RNA was extracted from follicular fluid samples which were already collected during oocyte retrieval and were kept in deep freezer at $-40 \dot{C}$ until time of micro RNA measurement. Isolation of miRNA of follicular fluid samples was carried out using a cell free specific kit, the miR Neasy Serum/ Plasma kit (Qiagen, German town, MD). Input volume of each follicular fluid sample for extraction was two hundred microliters $(200 \mu 1)$. The miR 39 mimic was added to each extraction as an internal spike in control using miRNeasy Serum/ Plasma Spike-In Control (Qiagen, German town, MD).The concentration of extracted miRNA was quantified using magnetic induction cycler (mic)/Australia. The method of miRNA extraction, reverse transcription, preamplification reaction, Quantitative Real-Time PCR for (Gene Expression Analysis) of miRNA predicted targets all were done according to instructions of the manufacturer.

The data displayed by mic qRT- PCR was given as CT (cycle threshold) value for each sample of follicular fluid. CT value referred to the number of amplification cycles required for fluorescent signal to exceed the background level. This means that are inversely proportional to the amount of micro RNA in the sample, so low CT level indicates high expression of miRNA in the sample and vice versa. miRNA with CT value more than 40 was considered not expressed in this study (Deo et al., 2011).

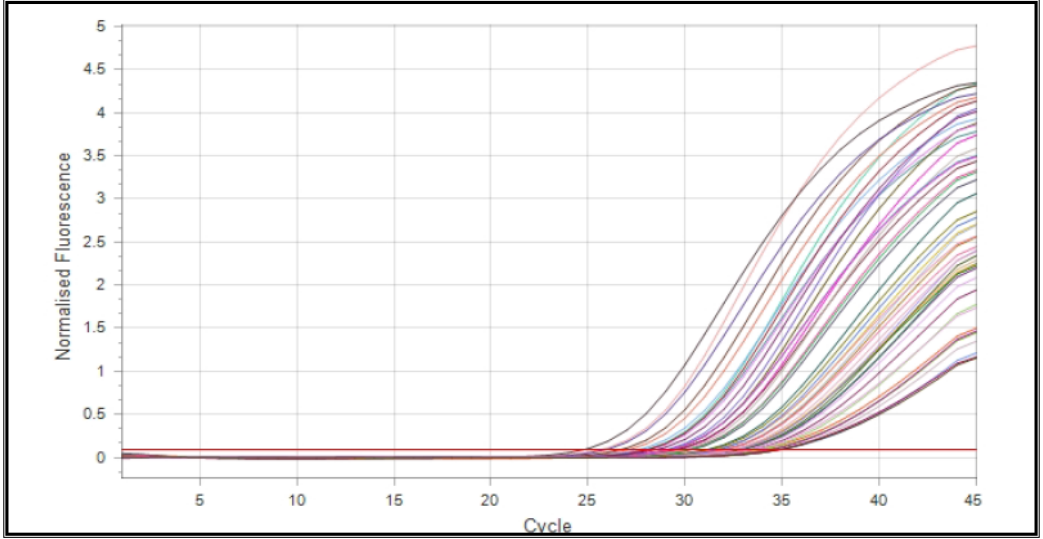

Figure 2:-Results of follicular fluid miRNA-320 generated by mic PCR version 2.4.0

Results:

The results of the present study were based on the analysis of a comparative study design with follicular fluid, oocyt and embryo of the first follicle retrieved. Ten patients were excluded after assessment of fertilization from measurement of miRNA- 320 which was measured in sixty one (61) samples only. These general parameters for all females included in the present study were summarized in table (1). 
Table 1:-Clinical parameters for all infertile females included in the study

\begin{tabular}{|c|c|c|}
\hline \multicolumn{2}{|c|}{ Variable } & Results \\
\hline Age (yr) & mean \pm SEM & $\mathbf{3 3 . 0} \pm 0.67$ \\
& Range & $\mathbf{2 0 - 4 3}$ \\
\hline BMI $\left(\mathrm{kg} / \mathrm{m}^{2}\right)$ & mean \pm SEM & $\mathbf{2 8 . 9 1 \pm 0 . 6 6}$ \\
& Range & $\mathbf{2 1 - 5 0}$ \\
\hline & mean \pm SEM & $\mathbf{7 . 7 \pm 0 . 4 5}$ \\
\hline & Range & $\mathbf{1 - 2 0}$ \\
\hline Duration of infertility (yr) & & $\mathbf{7 3 . 2 4 \%}$ \\
\hline \multicolumn{2}{|c|}{ Primary infertility (\%) } & $\mathbf{2 6 . 7 6 \%}$ \\
\hline \multicolumn{2}{|c|}{ Secondary infertility (\%) } & $\mathbf{7 6 . 0 6 \%}$ \\
\hline
\end{tabular}

The indications for IVF- ICSI were classified into ovulatory problems (36.61\%), tubal factors $(7.04 \%)$, male factors (with exception of those with azoospermia) (29.58\%) and unexplained infertility (26.76\%). The percentage of these factors was shown in figure 3 .

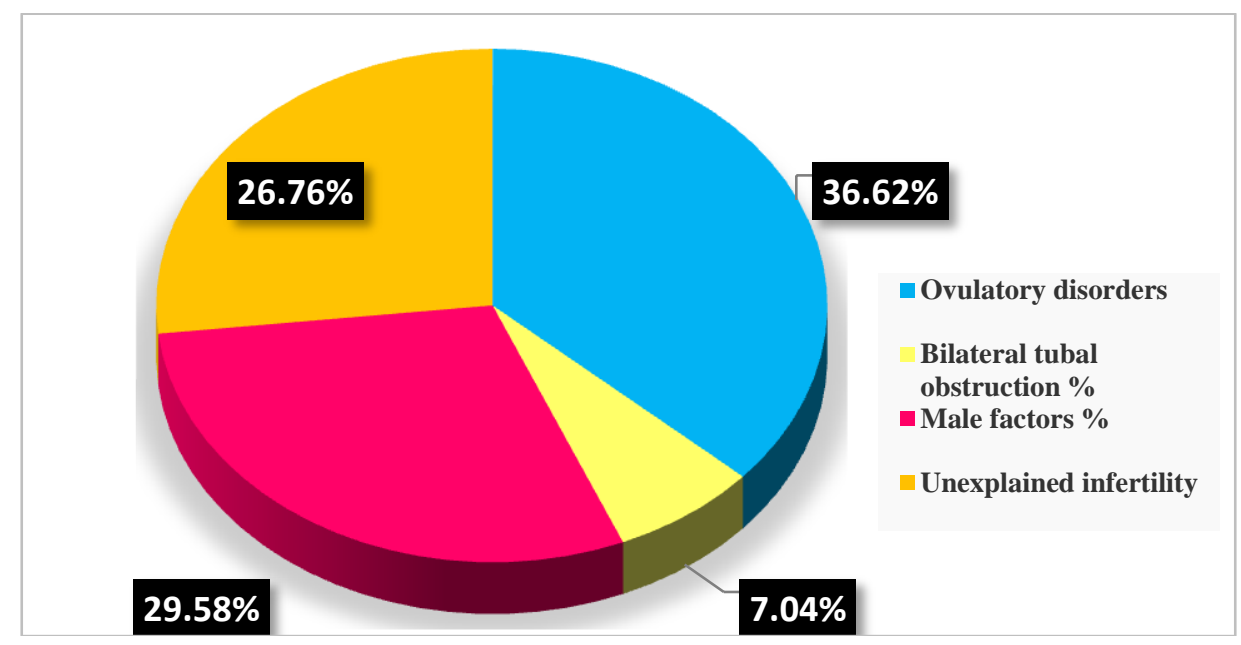

Figure 3: indications of IVF-ICSI in all infertile females included in the study Correlation of miRNA- 230 level in follicular fluid with clinical and ICSI parameters:

The statistical analysis showed no significant correlation between miRNA- 320 levels in follicular fluid and age, BMI, number of dominant follicles, number of oocytes retrieved, number of metaphase I, metaphase II, germinal vesicle, abnormal oocytes and number of fertilized oocytes (2PN) as shown in table (2)

Table 2:-correlation of miRNA- 320 with clinical and ICSI parameters

\begin{tabular}{|l|c|c|}
\hline \multirow{2}{*}{ Parameters (n=61) } & \multicolumn{2}{|c|}{ miRNA-320 } \\
\cline { 2 - 3 } & r & p \\
\hline Age (years) & 0.078 & $0.551^{\mathrm{NS}}$ \\
\hline BMI $\left(\mathrm{kg} / \mathrm{m}^{2}\right.$ ) & -0.200 & $0.122^{\mathrm{NS}}$ \\
\hline No. of dominant follicles & -0.022 & $0.864^{\mathrm{NS}}$ \\
\hline No. of oocytes retrieved & 0.006 & $0.965^{\mathrm{NS}}$ \\
\hline No. of Metaphase II oocytes (MII) & -0.011 & $0.931^{\mathrm{NS}}$ \\
\hline No. of Metaphase I oocytes (MI) & -0.093 & $0.478^{\mathrm{NS}}$ \\
\hline No. of Germinal vesicle (GV) & -0.023 & $0.858^{\mathrm{NS}}$ \\
\hline No. of Abnormal oocytes & 0.199 & $0.124^{\mathrm{NS}}$ \\
\hline No. of Fertilized oocytes (2PN) & -0.078 & \multicolumn{2}{|c|}{0.548} \\
\hline
\end{tabular}

NS: Not significant 


\section{Mirna-320 and embryo quality:-}

Of the selected seventy one oocytes, there were seven immature oocytes (GV stage or MI stage) and three abnormal oocytes, and these were excluded from the following ICSI treatment. Six of them were undergoing agonist protocol and four were undergoing antagonist protocol. These 61 follicular fluid samples and matched mature MII oocytes with normal fertilization outcome were used to identify miRNA-320 associated with oocyte/embryo development potential.

Embryo qualities of the selected oocytes were assessed three days after ICSI and were classified into two groups according to their morphological criteria. The first group consists of those with good quality embryos which have six or more blastomeres of equal size and with fragmentation 10-25\% (Grade I and II), while the other group consists of those with bad quality embryos (less than six blastomeres unequal size with fragmentation more than 25\%). The miRNA-320 was measured in the follicular fluid of the selected oocytes by using polymerase chain reaction (PCR) and their levels were compared to their embryos' quality.

hsa- miRNA-320 levels in follicular fluid samples were compared to their embryos' quality. It was found that hsahsa-miRNA-320 level was 47.02 \pm 6.84 in follicular fluid samples of good quality embryo which were present in 36 infertile females, and $3.12 \pm 0.95$ in other 25 samples which were related to bad quality embryo. Statistical analysis showed highly significant difference $(\mathrm{p}<0.001)$ in the expression level of hsa-miRNA-320 between good and bad quality embryos as shown in table (4-16).

The hsa-miRNA-320 levels were recorded in long agonist treated group. The level of hsa-miRNA-320 in follicular fluid samples of good quality embryos within the group was $42.15 \pm 6.49$, while the level of hsa-miRNA level in follicular fluid samples of bad quality embryos within the same group was $2.51 \pm 1.36$. There was highly significant difference $(\mathrm{p}<0.001)$ in the level of hsa-miRNA-320 between the good and bad quality embryos in long agonist treated group.

The hsa-miRNA-320 levels were estimated in antagonist treated group. The level of hsa-miRNA-320 in follicular fluid samples of good quality embryos within the group was $54.68 \pm 14.48$, while the level of hsa-miRNA level in follicular fluid samples of bad quality embryos within the same group was $4.42 \pm 0.57$. There was significant difference $(\mathrm{p}<0.05)$ in the level of hsa-miRNA-320 between the good and bad quality embryos in antagonist treated group.

On the other hand the levels of hsa-miRNA-320 in the follicular fluid were compared between agonist and antagonist groups and the statistical analysis revealed no significant difference ( $>0.05)$ between them whether for good or bad quality embryos as summarized in table 3 .

Table 3:-Relation between miRNA-320 levels and embryo quality in long agonist (G1) and antagonist treated groups (G2).

\begin{tabular}{|c|c|c|c|c|}
\hline \multirow{2}{*}{ Embryo quality } & \multicolumn{2}{|c|}{ Type of protocol } & Total & P-Value \\
\cline { 2 - 4 } & $\begin{array}{c}\text { hsa-miRNA-320 } \\
\text { (agonist protocol) }\end{array}$ & $\begin{array}{c}\text { hsa-miRNA-320 } \\
\text { (antagonist protocol) }\end{array}$ & & \\
\hline $\begin{array}{c}\text { Good quality embryos } \\
\text { (mean } \pm \text { SEM) }\end{array}$ & $\begin{array}{c}42.15 \pm 6.49 \\
(\mathrm{n}=22)\end{array}$ & $\begin{array}{c}54.68 \pm 14.48 \\
(\mathrm{n}=14)\end{array}$ & $\begin{array}{c}47.02 \pm 6.84 \\
(\mathrm{n}=36)\end{array}$ & $0.358 \mathrm{NS}$ \\
\hline $\begin{array}{c}\text { Bad quality embryos } \\
\text { (mean } \pm \text { SEM) }\end{array}$ & $\begin{array}{c}2.51 \pm 1.36 \\
(\mathrm{n}=17)\end{array}$ & $\begin{array}{c}4.42 \pm 0.57 \\
(\mathrm{n}=8)\end{array}$ & $\begin{array}{c}3.12 \pm 0.95 \\
(\mathrm{n}=25)\end{array}$ & $0.380 \mathrm{NS}$ \\
\hline Total & $\begin{array}{c}24.87 \pm 4.86 \\
(\mathrm{n}=39)\end{array}$ & $\begin{array}{c}36.4 \pm 10.51 \\
(\mathrm{n}=22)\end{array}$ & $\begin{array}{c}29.02 \pm 4.9 \\
(\mathrm{n}=61)\end{array}$ & $0.262 \mathrm{NS}$ \\
\hline P-Value & $<0.001^{* *}$ & $0.017^{*}$ & $<0.001^{* *}$ & \\
\hline
\end{tabular}

$\mathrm{P}<0.05$ : significant, $* * \mathrm{P}<0.01$ : Highly significant, NS: Not significant.

\section{Statistical analysis:-}

Numeric data were displayed as mean \pm standard error of mean (SEM), and the comparison between the two study groups using unpaired t-test. While categorical data were displayed as frequency and percentage and the comparisons were done using Fisher exact test and chi square test. The level of significance (p value) less than 0.05 considered as significant and less than 0.001 considered as high significant difference. The software used to do the 
statistical analyses was SPSS-Version 23 and Microsoft excel 2016 to measure the effects of difference factors in study parameters.

\section{Discussion:-}

Forty five million couples were affected by infertility in 2010, (Mascarenhas et al., 2012), and this number is expected to rise as child-bearing is increasingly delayed. More than 5 million children have been born through assisted reproduction (Mansour et al., 2014). Since the early 1980s, GnrH agonists were introduced for the prevention of a premature LH rise during ovarian stimulation. Gonadotrophin-releasing hormone (GnrH) antagonists were used for ovarian stimulation in ART during the last decade (Blockeel et al., 2012).

The present study was designed to compare the effect of protocol type (long agonist versus antagonist) on results of IVF-ICSI using molecular marker (miRNA- 320) which is known to be related to embryo quality.

Although the number of in vitro fertilization (IVF) procedures is increasing with time, the resulting pregnancy rates are still far less than desirable. It has been recorded that only about $30 \%$ of IVF cycles result in a pregnancy (Mansour et al., 2014).

Recently, it has become more important to develop methods that evaluate both implantation potential and individual embryo quality in order to make easier selection of embryo that is most likely to end with a successful pregnancy and healthy child. Rapid, accurate and reliable techniques for optimum embryo selection prior to transfer clearly have the potential to increase efficiency and safety of IVF, increase overall pregnancy success rate, and promote long-term health of the children born from these procedures (Tscherner $\mathrm{rt}$ al. 2015).

In the present study a correlation was done between miRNA- 320 levels in follicular fluid and age, BMI, number of dominant follicles, number of oocytes retrieved, number of metaphase I, metaphase II, germinal vesicle, abnormal oocytes and number of fertilized oocytes (2PN). The statistical analysis showed no significant correlation between them. This indicated that these parameters have no effect on the level of miRNA- 320.

Also miRNA- 320 levels were compared between the different grades of resulting embryos in the two tested groups and statistically highly significant difference was observed in all females this difference was also significant and highly significant when comparison was done between good and bad quality embryos in the two tested groups. These results are in accordance with that of previous studies which provided the first evidence that miRNAs in human follicular fluid are indicative of and can influence embryo quality (Feng et al., 2015). Another studies found that the proportions of mouse MII oocytes that developed into 2-cell and blastocyst-stage embryos were strongly affected by knockdown of miR-320, and this further indicated that miR-320 plays an important role in embryo development potential.

Also it was found that knocking down expression of miR-320 in mouse MII oocytes by injecting its inhibitor oligonucleotide. Most of embryos injected with miR-320 inhibitor- arrested at the 2-cell stage and only a few proceeded to develop into blastocysts indicating that miR-320 is essential for embryonic development. Thus the relation between the miR-320 expression level in follicular fluid and embryonic development was supported in ICSI patients as well as the in vitro fertilization and cultivation of mouse oocytes (Feng et al., 2015).

The last comparison was done between the level of miRNA-320 in the agonist and antagonist treated groups and showed no significant difference and this indicates that the effect of different protocols on embryo quality by using molecular marker (miRNA- 320) which may be used as non invasive method for embryo selection for transfer. These findings are in accordance with those of previous studies (Catt.2015; Santonocito et al., 2014; Scott et al., 2005) 


\section{References:-}

1 Blockeel C., Devroey P. Optimisation of the follicular phase in IVF/ICSI. FVV in ObGyn. $2012 ; 4$ (3): $203-$ 212

2 Catt J. Effect of Ovarian Stimulation Protocols on Oocyte and Embryo Quality. In: Ghumman S. (eds) Principles and Practice of Controlled Ovarian Stimulation in ART. New Delhi: Springer, 2015.chapter 36. Pp.558-569

3 Cupisti S, Dittrich R, Mueller A, Strick R, Stiegler E, Binder H, Beckmann M. W., Strisse P.Correlations between anti-mullerian hormone, inhibin B, and activin A in follicular fluid in IVF/ICSI patients for assessing the maturation and developmental potential of oocytes. Eur J Med Res. 2007; 12, 604.

4 da Silveira JC, Veeramachaneni DN, Winger QA, Carnevale EM, Bouma GJ. Cell-secreted vesicles in equine ovarian follicular fluid contain miRNAs and proteins: a possible new form of cell communication within the ovarian follicle. Biol Reprod. 2012; 86:71.

5 Diez-Fraile A, Lammens T, Tilleman K, Witkowski W, Verhasselt B, De Sutter P, Benoit Y, Espeel M, D'Herde K. Age-associated differential microRNA levels in human follicular fluid reveal pathways potentially determining fertility and success of in vitro fertilization. Hum Fertil (Camb) . 2014; 17, 90.

6 Feng R, Sang K, Zhu Y, Fu W, Liu M, Xu Y, Shi H, Xu Y, Qu R, Chai R, Shao R, JinL, He L, Sun X\& Wang L.. MiRNA-320 in the human follicular fluid is associated with embryo quality in vivo and affects mouse embryonic development in vitro. Scientific report. 2015; 5: 8689. P1-9

7 Kim V.N. MicroRNA biogenesis: Coordinated cropping and dicing. Nat. Rev. Mol. Cell Biol. $2005 ; 6: 376$.

8 Lédée N, Lombroso R, Lombardelli L, Selva J, Dubanchet S, Chaouat G, Frankenne F, Foidart JM, Maggi E, Romagnani S, Ville Y, Piccinni MP. Cytokines and chemokines in follicular fluids and potential of the corresponding embryo: the role of granulocyte colony-stimulating factor. Hum Reprod . 2008; $23,2001$.

9 Macklon NS, Stouffer RL, Giudice LC \& Fauser BC. The science behind 25 years of ovarian stimulation for in vitro fertilization. Endocrine Reviews. 2006; 27 170-207Deo et al., 2011

10 Mansour, R., Ishihara, O., Adamson, G. D., Dyer, S., de Mouzon, J., Nygren, K.- G., Sullivan, E. and ZegersHochschild, F. (2014). International committee formonitoring assisted reproductive technologies world report: assisted reproductive technology 2006. Hum. Reprod. 29, 1536-1551.

11 Mascarenhas, M. N., Flaxman, S. R., Boerma, T.,Vanderpoel, S. and Stevens, G. A.. National, regional, and global trends in infertility prevalence since 1990: a systematic analysis of 277 health surveys. PLoS Med. 2012; 9, e1001356.

12 Santonocito, M. Santonocito M, Vento M, Guglielmino MR, Battaglia R, Wahlgren J, Ragusa M, Barbagallo D, Borzì P, Rizzari S, Maugeri M, Scollo P, Tatone C, Valadi H, Purrello M, Di Pietro C.. Molecular characterization of exosomes and their microRNA cargo in human follicular fluid: bioinformatic analysis reveals that exosomal microRNAs control pathways involved in follicular maturation. Fertil Steril . 2014; 102, 1751.

13 Scott, A. Finn, V. Cardone, I. Hardy, J. Hill, M. Hosseinzadeh. Fertility Centers of New England, Reading, MA. Stimulation Protocol Does not Affect Embryo Quality or Pregnancy Rates: A Prospective Study. L. Fertility \&Sterility. 2005; Vol. 84, Suppl 1.

14 Sohel MM, Hoelker M, Noferesti SS, Salilew-Wondim D, Tholen E, Looft C, et al. Exosomal and nonexosomal transport of extra-cellular microRNAs in follicular fluid: implications for bovine oocyte developmental competence. PLoS One . 2013;8:e78505.

15 Sonderegger, S., Pollheimer, J. \& Knofler, M. Wnt signalling in implantation, decidualisation and placental differentiation--review. Placenta . 2010;31, 839.

16 Su L, Zhao S, Zhu M, Yu M. Differential expression of microRNAs in porcine placentas on Days 30 and 90 of gestation. Reproduction, Fertility and Development. 2010; 22:1175-1182.

17 Tscherner A, Stalker L and LaMarre J. Is there a small RNA fingerprint of embryo quality and health inspent IVF media? Fertility Genetics Magazine. $2015 \cdot$ Volume $1 \bullet P 6-8$

18 Wahid F, Shehzad A, Khan T, Young Kim Y. MicroRNAs: Synthesis, mechanism, function, and recent clinical trials. Biochimica et Biophysica Acta. 2010; 1803:1231-1243. 